\section{European \\ Surgical Research}

\title{
Authors List
}

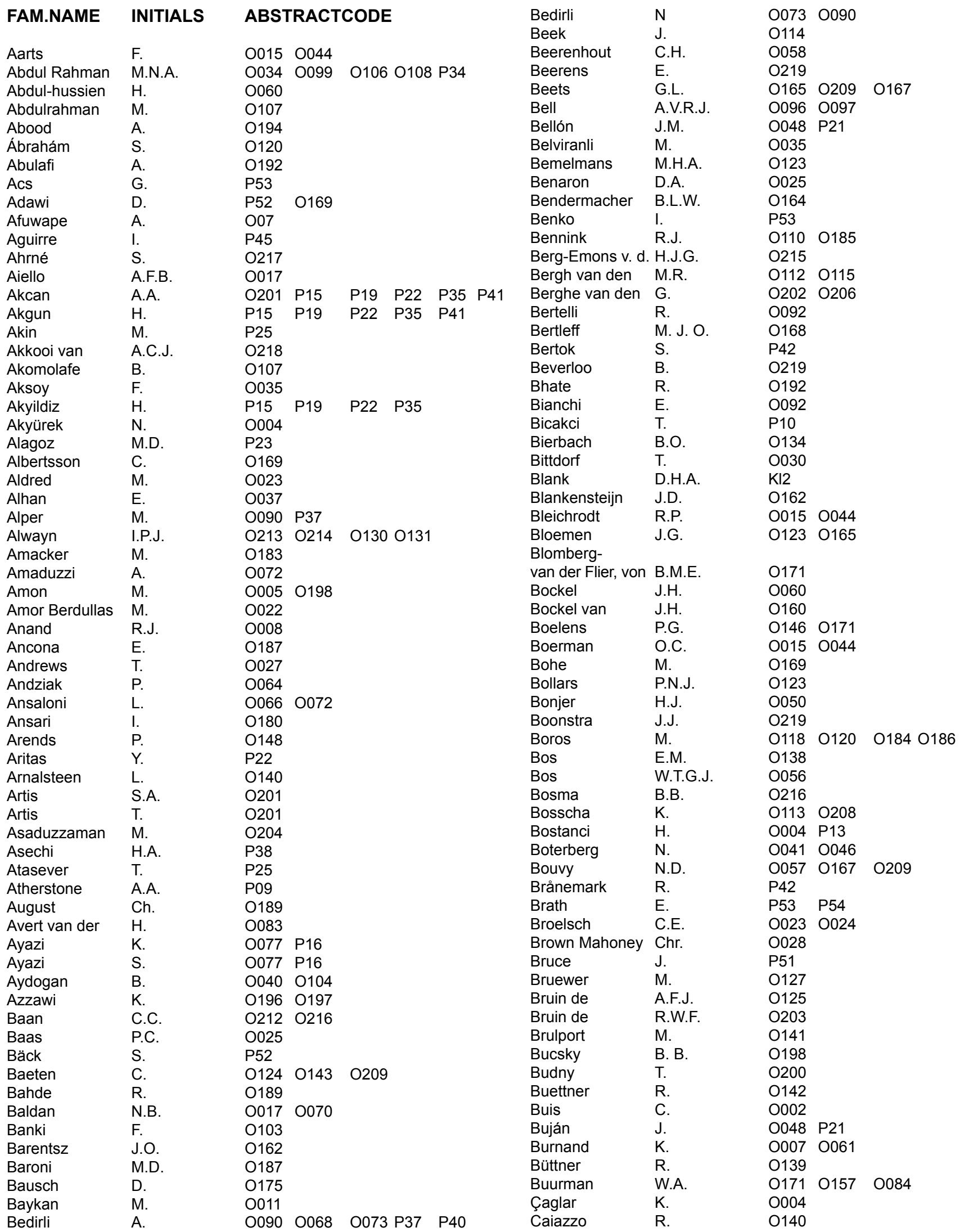




\begin{tabular}{|c|c|c|c|c|c|c|c|c|c|}
\hline Cainzos & M. & $\mathrm{O} 022$ & & & Dirksen & C.D. & 0097 & & \\
\hline Cakala & M. & 0117 & 0176 & & Dobberahn & V. & O089 & O018 & \\
\hline Can & M. & $\mathrm{O} 036$ & & & Dogan & M. & P25 & & \\
\hline Canal & A.C. & 0078 & O155 & & Dokanakoglu & O. & 0102 & & \\
\hline Canda & A.E. & O101 & 0102 & O038 O040 O104 & Dold & S.D. & O220 & O205 & $\mathrm{O} 221$ \\
\hline Carbajo & M. & 0173 & & & Dominiak & A. & 0098 & 0137 & \\
\hline Carter & F. & $\mathrm{O} 053$ & & & Doorn van & L. J. & 0168 & & \\
\hline Carvalho & C. & 0031 & & & Doornebosch & P. & $\mathrm{O} 210$ & & \\
\hline Catena & F. & $\mathrm{O} 066$ & O072 & & Doorschodt & B. & 0139 & & \\
\hline Cavallari & G. & 0092 & & & Dósa & E. & O158 & & \\
\hline Cavallo & J.A. & $\mathrm{O} 008$ & & & Dubowski & T.D. & 0008 & & \\
\hline Cearra & I. & O178 & & & Duijff & J.W. & 0049 & O067 & \\
\hline Ceelen & W.P. & 0041 & 0046 & 0047 & Dunaway & D. & O195 & & \\
\hline Cehreli & $\mathrm{R}$. & 0038 & 0101 & & Dungen van den & J.J.A.M. & $\mathrm{O} 056$ & & \\
\hline Cengiz & C.E. & 0037 & & & Durlik & M. & 0064 & O079 & 0137 \\
\hline Cervenak & L. & O158 & & & Dwars & B.J. & P55 & & \\
\hline Cetinkaya & O. & $\mathrm{O} 040$ & & & Earl & $\mathrm{H}$. & O196 & & \\
\hline Chan & F.C. & $\mathrm{O} 078$ & 0151 & & Ebrahimian & $\mathrm{T}$. & O163 & 0006 & \\
\hline Chand & M. & $\mathrm{O} 027$ & & & Echevarria & J.J. & P45 & & \\
\hline Chaudhari & A. & 0111 & & & Echten & & & & \\
\hline Chen & S. & O069 & & & Deckert van & G. & 0139 & & \\
\hline Chetter & I.C. & $\mathrm{O} 034$ & O099 & O106 0107 O108 P34 & Edu & M. & O009 & & \\
\hline Chhabra & D. & 0111 & & & Eertwegh & & & & \\
\hline Chia & H.L. & 0150 & & & van den & A.J.M. & O179 & & \\
\hline Christie & L. & 0053 & & & Ege & B. & P27 & & \\
\hline Cianciavicchia & D. & $\mathrm{O} 092$ & & & Eggermont & A.M.M. & 0147 & O159 & O181 O210 KI4 \\
\hline Cikirikcioglu & M. & 0133 & & & Eijck van & C.H.J. & O222 & $\mathrm{O} 074$ & $\mathrm{O} 211$ \\
\hline Clarke & A.D. & $\mathrm{O} 027$ & & & Eipel & C. & O082 & O094 & 0141 \\
\hline Cleutjens & J.P. & 0059 & & & Ekice & $\mathrm{T}$. & 0102 & & \\
\hline Coccolini & F. & O066 & & & Ekinci & O. & O073 & & \\
\hline Cogliandolo & A. & O045 & & & Ekser & B.E. & 0017 & $\mathrm{O} 070$ & \\
\hline Coker & A. & P11 & & & El Kased & A.F. & 0166 & & \\
\hline Collinson & P. & O192 & & & El-Bahrawy & M. & O039 & & \\
\hline Coolsen & M.M.E & 0123 & & & Elbeg & $\mathrm{S}$. & 0105 & & \\
\hline Cormack & G. & $\mathrm{O} 078$ & & & Eleftheriadis & E. & P26 & P28 & P29 \\
\hline Corrales & c. & P21 & & & Ellger & B. & O202 & O206 & \\
\hline Coughlin & P. & O099 & 0106 & 01070108 & Elsebae & M.A. & 0012 & O065 & 01210051 \\
\hline Crezee & $\mathrm{H}$. & 0114 & & & Elst van der & M. & 0129 & & \\
\hline Crom de & R. & O161 & 0163 & 0006 & Emans & P.J. & O209 & O167 & \\
\hline Cruz & S.O. & 0133 & & & Eng & C. & O023 & O024 & \\
\hline Cserepes & B. & O191 & & & Engel van den & $\mathrm{s}$. & O203 & & \\
\hline Csontos & C. & P44 & & & Engelen & S.E.M. & O165 & & \\
\hline Cuellar & A. & P45 & & & Engin & D. & P24 & & \\
\hline Cuesta & M.A. & 0112 & 0115 & $\mathrm{O} 171$ & Enomoto & Y. & 0013 & P02 & \\
\hline Cuschieri & A. & $\mathrm{O} 053$ & & & Ent van der & F.W. & 0096 & O097 & \\
\hline Cwikla & J. & $\mathrm{O} 117$ & 0177 & & Entz & L. & O158 & & \\
\hline Czóbel & $\mathrm{M}$. & 0184 & & & Érces & D. & 0118 & & \\
\hline Dadoukis & D. & P29 & & & Erdem & O. & P24 & & \\
\hline Dahlmans & A.A.H.R. & O164 & & & Erdmann & $\mathrm{J}$. & $\mathrm{O} 211$ & & \\
\hline D'Alessandro & L. & $\mathrm{O} 072$ & & & Erdogan & A.R. & P22 & & \\
\hline Dall'Olmo & L. & 0187 & & & Erdogan & D. & 0032 & & \\
\hline Dam van & G.M. & $\mathrm{O} 025$ & O057 & 0123 & Erenoglu & B. & O011 & & \\
\hline Damme Van & N. & 0041 & 0046 & & Ernst & M.F. & O208 & & \\
\hline Dankelman & J. & 0129 & 0132 & & Eros & G. & O184 & & \\
\hline Davaie & M. & P16 & & & Erp van & A. & O028 & & \\
\hline Dazzo & E. & 0187 & & & Ersin & S. & P10 & & \\
\hline Debaveye & Y. & O202 & O206 & & Ersoy & E. & P27 & & \\
\hline Debrah & S.A. & 0014 & & & Esawi & M. & 0166 & & \\
\hline Dejong & C.H. & O084 & 0123 & 0146 & Esin & H.E. & O201 & & \\
\hline Dekken van & $\mathrm{H}$. & $\mathrm{O} 219$ & & & Estrada & J.J. & O069 & & \\
\hline Delawi & D. & O028 & & & Eussen & E. & $\mathrm{O} 164$ & & \\
\hline DeMeester & S.R. & O103 & & & Evans & R.H. & O195 & & \\
\hline DeMeester & T.R. & 0103 & & & Everts & P.A.M. & 0028 & & \\
\hline Demirel & G. & $\mathrm{O} 036$ & & & Ezzat & $\mathrm{H}$. & 0166 & & \\
\hline Demirkiran & A. & $\mathrm{O} 212$ & $\mathrm{O} 216$ & & Ezzat & H.E. & $\mathrm{O} 012$ & 0051 & \\
\hline Derczy & $\mathrm{K}$. & P42 & & & Fabris & L.M.F. & $\mathrm{O} 017$ & 0070 & \\
\hline Deutz & N.E.P. & 0146 & & & Famà & F.W. & 0045 & & \\
\hline Dhert & W. & O028 & & & Fanaie & A. & P04 & & \\
\hline Di Saverio & S. & O066 & O072 & & Ferencz & A. & O191 & & \\
\hline Diepenhorst & G.M.P. & O119 & & & Ferencz & Á. & 0120 & & \\
\hline Dijck van & J. & O019 & & & Ferencz & S. & P42 & P44 & \\
\hline Dijk van & P.G. & 0049 & & & Ferenschild & F.T.J. & 0147 & & \\
\hline Dikmen & $\mathrm{K}$. & O105 & & & Fernandez & M.C.F. & O145 & & \\
\hline Diks & J. & 0171 & & & Fibbe & W.E. & O160 & & \\
\hline Dimitriou & C.A. & 0062 & & & Fierens & N. & 0046 & 0047 & \\
\hline Dinjens & W.N.M. & O222 & O219 & & Fini & M. & 0066 & & \\
\hline
\end{tabular}




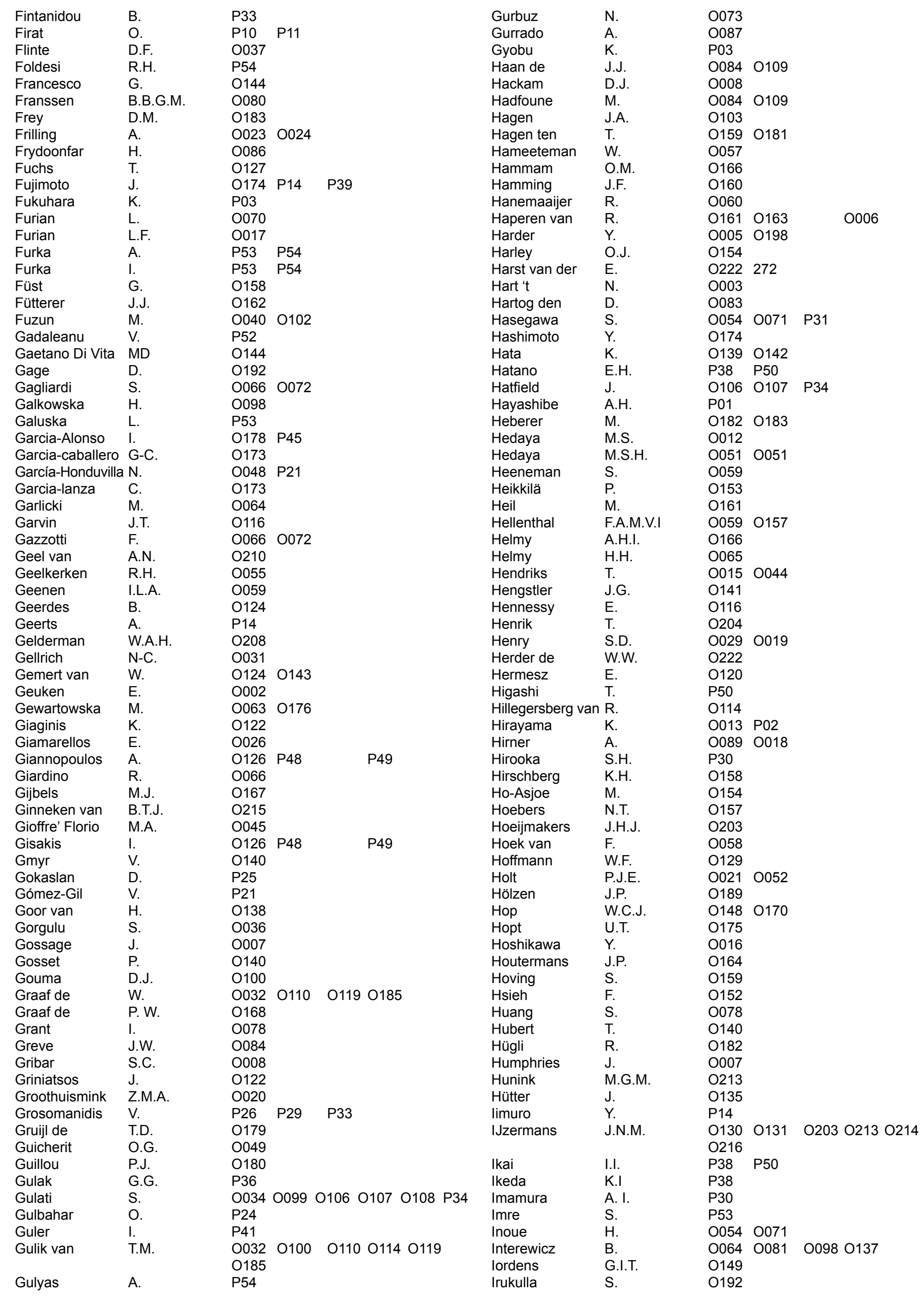




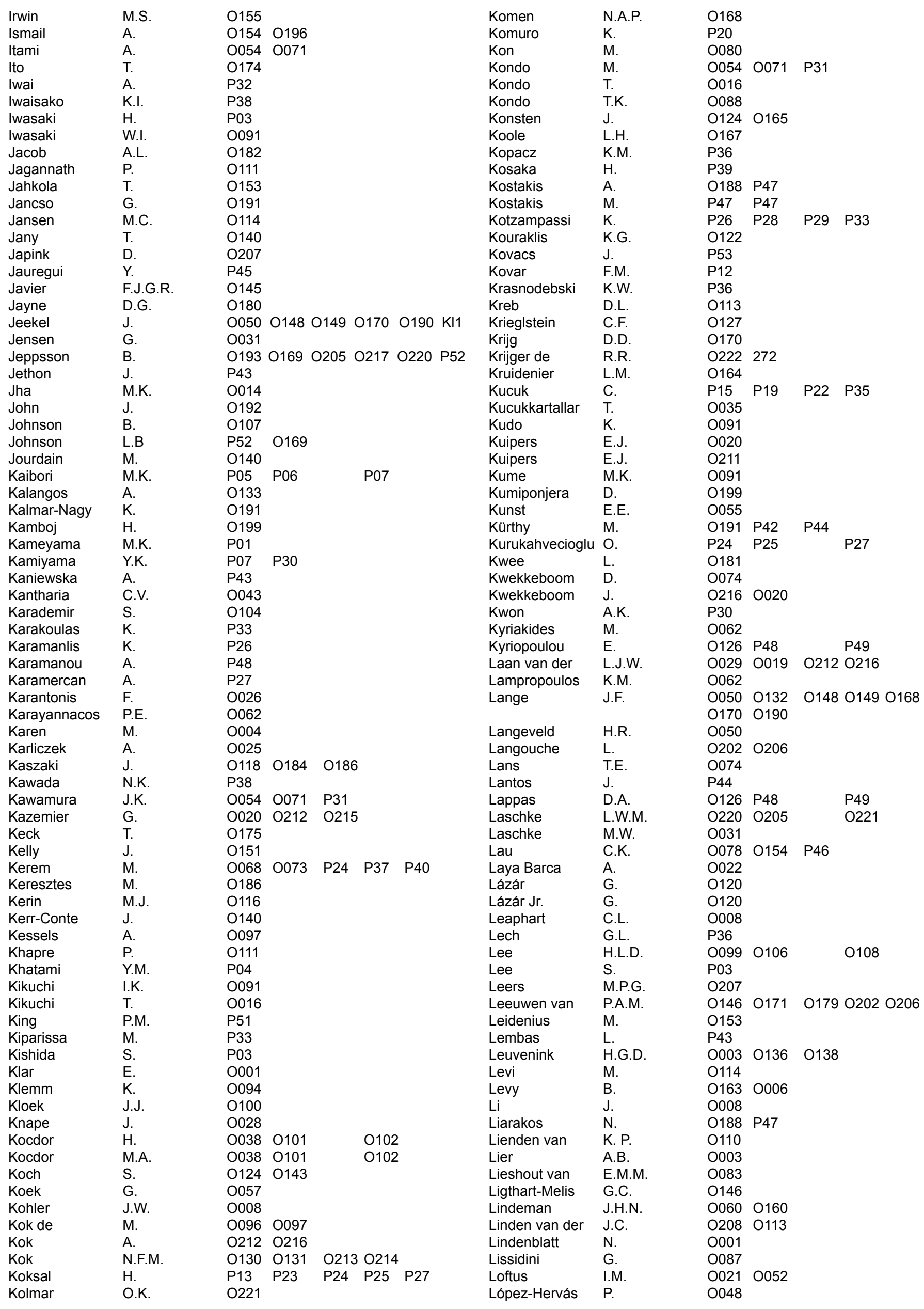




\begin{tabular}{|c|c|c|c|c|c|c|c|c|c|c|c|}
\hline Lubbers & $\mathrm{T}$. & O084 & \multicolumn{2}{|l|}{0109} & Morita-Fujimura & Y. & O219 & & & & \\
\hline Lubics & A. & O191 & & & Motoyama & S.M. & 0093 & & & & \\
\hline Luczak & M. & 0098 & & & Mouzopoulos & D. & P17 & & & & \\
\hline Luirink & M. & 0058 & & & Mouzopoulos & $\mathrm{G}$. & P17 & P18 & & & \\
\hline Luyer & M.D. & O084 & & & Muhs & B.E. & 0056 & & & & \\
\hline Maathuis & M.H.J. & 0003 & & & Muhtaroglu & S. & P19 & P41 & & & \\
\hline Machens & H.G.M. & O198 & & & Mülhaupt & $\mathrm{R}$. & 0031 & & & & \\
\hline Machowski & Z. & O098 & & & Murata & S.M. & O088 & & & & \\
\hline Makay & $\mathrm{O}$ & P10 & P11 & & Mutlu & F.M. & O201 & & & & \\
\hline Maksymowicz & M. & 0063 & & & Mutlukoca & $\mathrm{N}$ & P11 & & & & \\
\hline Malata & C.M. & O196 & & & Nagata & H.N. & P38 & & & & \\
\hline Malata & C.M. & 0152 & 0155 & 019401960199 & Nagatani & S. & P31 & & & & \\
\hline Man de & R.A. & 0020 & & & Nagayama & S. & O054 & 0071 & P31 & P32 & \\
\hline Manekeller & S. & 0003 & 0018 & O089 & Nagy & B. & P54 & & & & \\
\hline Maniega & R. & P45 & & & Nakagawa & Y.N. & 0093 & & & & \\
\hline Manjer & J. & 0169 & & & Nakajima & Y. & 0013 & P02 & & & \\
\hline Mantas & D. & 0026 & 0188 & P47 & Nakanishi & $\mathrm{K}$. & P39 & & & & \\
\hline Margaritis & E. & 0188 & P47 & & Nap & M. & O207 & & & & \\
\hline Marin & $\mathrm{H}$. & P45 & & & Nardo & B. & 0092 & & & & \\
\hline Marion van & R. & $\mathrm{O} 219$ & & & Narita & M.N. & P38 & & & & \\
\hline Marsman & H.A. & 0100 & & & Nash & G.F. & 0027 & & & & \\
\hline Martinez Castro & J. & 0022 & & & Nasr & M.A.N. & 0051 & & & & \\
\hline Martinez- & & & & & Navsaria & P.H. & 0009 & & & & \\
\hline Astorquiza & $\mathrm{T}$. & 0178 & & & Nemeth & $\mathrm{N}$. & P53 & P54 & & & \\
\hline Maruszynski & M. & 0176 & & & Németh & I. & 0186 & & & & \\
\hline Mason & R.J. & 0103 & 0069 & & Neri & F.M. & 0092 & & & & \\
\hline Matsui & K.M. & P07 & & & Nicol & A.J. & 0009 & & & & \\
\hline Matsumura & Y. & 0016 & & & Nicolai & S.P.A. & 0164 & & & & \\
\hline Matsuo & R.M. & 0088 & & & Niessen & H.W. & 0119 & & & & \\
\hline Matteo Arcara & MD & O144 & & & Nieuwenhuizen & J. & 0148 & 0170 & 0190 & & \\
\hline Matthijsen & R.A. & 0157 & & & Nijveldt & R.J. & P55 & & & & \\
\hline Mattsson & S. & 0193 & & & Nikiteas & $\mathrm{N}$. & 0026 & P47 & & & \\
\hline Matziolis & G. & 0030 & & & Ninci & $\mathrm{E}$. & 0161 & & & & \\
\hline McCallum & I.J.D. & P51 & & & Nishizawa & S. & P03 & & & & \\
\hline McCollum & P.T. & 0034 & O099 & O106 O107 O108 P34 & Noda & M. & 0016 & & & & \\
\hline McGrath & D.R. & 0086 & & & Nomura & A. & 0054 & 0071 & P31 & & \\
\hline McNeill & R.E. & 0116 & & & Noorden van & C.J.F. & 0076 & & & & \\
\hline Mearadji & A. & $\mathrm{O} 210$ & & & Ntziora & F.M. & P49 & & & & \\
\hline Mees & B.M.E. & 0161 & 0163 & 0006 & Nuessler & A.K. & 0141 & & & & \\
\hline Mees & S.T. & 0127 & & & Nuyttens & J.J.M.E. & 0125 & 0147 & & & \\
\hline Meijer & S. & 0112 & 0115 & 0179 & Obertop & $\mathrm{H}$. & 0074 & & & & \\
\hline Meijerink & M.R. & 0112 & 0115 & & Oertli & D. & 0182 & 0183 & & & \\
\hline Mekako & A. & 0034 & 0099 & 01060107 & Ofluoglu & E. & 0004 & 0068 & 0090 & 0 P37 & \\
\hline & & 0108 & P34 & & $\mathrm{Oh}$ & D. & 0103 & & & & \\
\hline Melenhorst & J. & 0124 & 0143 & & Ohiami-Masseron & $\mathrm{S}$. & P52 & & & & \\
\hline Melis-Lighthart & G.C. & 0171 & & & Ohkohchi & N.O. & 0088 & & & & \\
\hline Memis & L. & 0004 & & & Oishi & $\mathrm{H}$. & 0016 & & & & \\
\hline Mena del Rio & E. & 0022 & & & Ok & E. & P15 & P41 & & & \\
\hline Mendall & M.A. & 0192 & & & Okabe & $\mathrm{H}$. & 0054 & 0071 & & & \\
\hline Menger & M.D. & 0001 & 0005 & O031 O198 O205 & Okada & Y. & 0016 & & & & \\
\hline & & $\mathrm{O} 220$ & $\mathrm{O} 221$ & & Okoshi & $\mathrm{K}$. & P32 & & & & \\
\hline Menshykova & M. & 0030 & & & Oktay & G. & 0040 & & & & \\
\hline Mentes & B. & P13 & & & Okubo & Y.O. & P30 & & & & \\
\hline Meretoja & $\mathrm{T}$. & 0153 & & & Okumura & $\mathrm{T}$. & 0174 & & & & \\
\hline Metselaar & H.J. & $\mathrm{O} 215$ & 0019 & O020 O029 O212 & Okuno & M.O. & P30 & & & & \\
\hline & & 0215 & 0216 & & Olde Damink & S.W.M. & 0123 & & & & \\
\hline Meyenfeldt & M.F. & 0096 & 0097 & & Olszewski & W.L. & 0063 & 0064 & 0079 & 0081 & 0098 \\
\hline Michail & O.M. & 0122 & & & & & 0117 & 0137 & 0177 & 0176 & P36 \\
\hline Michail & P. & 0122 & & & Omur & O. & P11 & & & & \\
\hline Mihaescu & I. & 0193 & $\mathrm{O} 217$ & & Onen & Z. & P11 & & & & \\
\hline Miko & I. & P53 & P54 & & Ong & J.C.Y. & 0151 & & & & \\
\hline Miller & $\mathrm{N}$. & 0116 & & & Ono & Y.O. & 0091 & & & & \\
\hline Minguez & A. & 0173 & & & Orhan & D. & P23 & & & & \\
\hline Minh, Le & $\mathrm{K}$. & 0094 & & & Ortiz & D. & 0174 & & & & \\
\hline Minin & $\mathrm{E}$. & 0189 & & & Osorio & D. & 0173 & & & & \\
\hline Minor & $\mathrm{T}$. & 0003 & 0018 & 008901390142 & Osugi & H. & P03 & & & & \\
\hline Mitchell & J.R. & $\mathrm{O} 203$ & & & Ottochian & B. & 0169 & & & & \\
\hline Mittlmeier & $\mathrm{T}$. & 0030 & 0082 & & Overdevest & E. & 0028 & & & & \\
\hline Miura & Y. & 0013 & P02 & & Ozaki & T.O. & P30 & & & & \\
\hline Mizuno & Y.M. & P31 & P32 & & Ozlu & O. & 0090 & & & & \\
\hline Modarai & B. & 0007 & & & Ozutemiz & O. & P10 & & & & \\
\hline Moers & C. & 0136 & & & Pacilè & V. & 0092 & & & & \\
\hline Molenkamp & B.G. & 0179 & & & Palásthy & Z. & 0118 & & & & \\
\hline Molin & G. & $\mathrm{O} 217$ & & & Paleolo & $\mathrm{E}$. & 0007 & & & & \\
\hline Moore & P.J. & 0027 & 81 & & Palmes & D. & 0095 & & & & \\
\hline Mori & Y. & 0174 & P32 & & Palomares & $\mathrm{T}$. & 0178 & P45 & & & \\
\hline
\end{tabular}




\begin{tabular}{|c|c|c|c|c|c|c|c|c|c|c|}
\hline Pantopoulou & A. & O188 & P47 & & Rutten & M.J.C.M. & O113 & & & \\
\hline Papachristodoulou & $A$. & O188 & P17 & P18 & Rydzewski & A. & O137 & & & \\
\hline Papalouka & V. & 0062 & & & Sachs & $\mathrm{L}$. & 0127 & & & \\
\hline Paramythiotis & D. & P26 & P29 & & Sadek & A.S. & O121 & & & \\
\hline Pasaoglu & $\mathrm{H}$. & 0004 & 0068 & O073 O090 O105 P37 & Sado & S. & 0016 & & & \\
\hline Pascual & G. & 0048 & & & Safioleas & M. & P17 & P18 & & \\
\hline Patel & D. & 0061 & & & Sagol & O. & O101 & O102 & & O104 \\
\hline Patka & P. & 0083 & & & Sahin & M. & 0011 & 0035 & P23 & \\
\hline Pattou & F. & 0140 & & & Sahin & $\mathrm{T}$. & 0004 & 0105 & & \\
\hline Pattyn & $\mathrm{N}$. & 0041 & 0046 & 0047 & Saint-Marc & O. & 0045 & & & \\
\hline Peeters & $\mathrm{N}$. & 0041 & 0046 & 0047 & Saito & T.S. & P07 & & & \\
\hline Pektok & $\mathrm{E}$. & 0133 & & & Saitoh & K. & 0013 & P02 & & \\
\hline Perrea & D. & 0026 & 0188 & P47 & Sakai & Y. & 0054 & 0071 & P31 & P32 \\
\hline Peto & K. & P53 & & & Sakrak & O. & O068 & 0073 & P40 & \\
\hline Peto & K. & P54 & & & Saliangas & K. & P26 & & & \\
\hline Petri & B.J. & $\mathrm{O} 222$ & & & Salman & B. & 0068 & O105 & P37 & P40 \\
\hline Petrosyan & M. & 0069 & & & Salvatore & & & & & \\
\hline Piccinni & G. & 0087 & & & Buscemi & M.S. & O144 & & & \\
\hline Pidoto & $\mathrm{R}$. & 0045 & & & San Cristobal & J. & 0178 & & & \\
\hline Pinna & A.D. & 0066 & $\mathrm{O} 072$ & & San Isidro & $\mathrm{R}$. & O178 & & & \\
\hline Piquard & A. & 0045 & & & Sapy & P. & P54 & & & \\
\hline Piras & G.L. & 0092 & & & Sarantopoulos & E. & 0126 & P49 & & \\
\hline Pittet & B. & 0005 & & & Saraymen & $\mathrm{R}$. & P35 & & & \\
\hline Plaats van der & A. & 0003 & & & Sare & M. & P40 & & & \\
\hline Planting & A.S.T. & 0125 & & & Sato & S. & O054 & 0071 & & \\
\hline Ploeg & R.J. & 0003 & 0136 & & Sato & T.S. & 0091 & 0093 & & \\
\hline Poley & J.W. & O211 & & & Scandalakis & P. & O126 & P48 & & P49 \\
\hline Poli & E. & O087 & & & Schaik van & P.M. & O208 & & & \\
\hline Polistena & A. & P52 & & & Schaper & W. & O161 & & & \\
\hline Poll van der & C.G. & 0146 & & & Scheltinga & M.R.M. & O058 & & & \\
\hline Poloniecki & J.D. & 0021 & 0052 & & Scheper & R.J. & 0179 & & & \\
\hline Ponniah & A.J. & O155 & O195 & & Schepers & $\mathrm{T}$. & 0083 & & & \\
\hline Porte & R.J. & 0002 & & & Schilling & M.K.S. & O220 & O205 & & O221 \\
\hline Portincasa & P. & 0087 & & & Schipper & D. & 0159 & & & \\
\hline Poulakou & M. & O188 & P47 & & Schipper & I.B. & 0083 & & & \\
\hline Poyraz & A. & O105 & P25 & & Schoen & M. & 0030 & & & \\
\hline Prezzi & D. & 0092 & & & Scholte & B.J. & 0029 & & & \\
\hline Prins & $\mathrm{R}$. & 0056 & & & Schoots & I. & O114 & & & \\
\hline Prohászka & Z. & O158 & & & Schramm & $\mathrm{R}$. & 0005 & & & \\
\hline Pruijt & J.F.M. & 0113 & & & Schreinemacher & M.H.F. & O209 & O167 & & \\
\hline Putte van de & N. & 0046 & & & Schultaz & H. & 0135 & & & \\
\hline Puviani & L. & 0092 & & & Schulz & T. & O156 & & & \\
\hline Racz & B. & 0191 & & & Schumacher & $\mathrm{H}$. & 0150 & & & \\
\hline Rácz & A. & 0118 & & & Schumacher & H.A. & P46 & & & \\
\hline Radovits & $\mathrm{R}$. & O135 & & & Schumacher & $\mathrm{R}$. & O183 & & & \\
\hline Rajaganeshan & $\mathrm{R}$. & 0180 & & & Schurink & G.W.H. & 0059 & 0157 & & \\
\hline Rakhorst & $\mathrm{G}$. & 0003 & & & Schuurman & A.H. & 0080 & & & \\
\hline Ramshorst van & $\mathrm{GH}$ & 0148 & O170 & & Scott & $\mathrm{N}$. & O180 & & & \\
\hline Rázga & Z. & 0186 & & & Seaward & $\mathrm{R}$. & 0199 & & & \\
\hline Regan & P. & 0151 & & & Segat & D. & O187 & & & \\
\hline Reglodi & D. & O191 & & & Sener & M. & O101 & & & \\
\hline Rens & J. & 0181 & & & Senninger & N. & O127 & & & \\
\hline Renwick & P. & 0107 & & & Seo & S.S. & P38 & P50 & & \\
\hline Reynaert & $\mathrm{H}$. & P14 & & & Serfozo & J. & P53 & & & \\
\hline Rezaeian & $\mathrm{F}$. & 0005 & & & Sevinc & A.I. & 0040 & & & \\
\hline Richards & $\mathrm{R}$. & 0195 & & & Sewgobind & V. & $\mathrm{O} 212$ & & & \\
\hline Richir & M.C. & 0171 & O202 & O206 & Seynhaeve & A. & O159 & & & \\
\hline Richter & S.R. & $\mathrm{O} 221$ & & & Shadmehr & M.B. & 0077 & & & \\
\hline Rigotti & P. & 0017 & $\mathrm{O} 070$ & & Shah & A. & O152 & & & \\
\hline Rijcken & E.J.M. & O127 & & & Shah & $\mathrm{R}$. & O111 & & & \\
\hline Rijk & P.C. & 0076 & & & Shawish & E. & 0039 & & & \\
\hline Rizk & H. & O166 & & & Shibata & S.S. & 0093 & & & \\
\hline Rödel & S.G.J & 0055 & & & Shimada & T. & P02 & & & \\
\hline Rodríguez & M. & 0048 & P21 & & Shimada & Y. & 0174 & P32 & & \\
\hline Rodriguez Couso & J.L. & O022 & & & Sietses & C. & O112 & 0115 & & \\
\hline Roest & H.P. & O203 & & & Sievert & K. & 0141 & & & \\
\hline Rogers & A.D. & P09 & & & Silvestre & J.S. & 0006 & 0163 & $\mathrm{KI} 3$ & \\
\hline Rongen & M.J. & O124 & & & Simcock & J. & O150 & 0155 & & \\
\hline Rosalia Patti & MD & O144 & & & Simpson & E. & P09 & & & \\
\hline Rosenthal & $\mathrm{R}$. & O182 & 0183 & & Simsek & A. & 0036 & & & \\
\hline Roth & E. & O191 & P42 & P44 & Sipka & S. & P53 & & & \\
\hline Rotter & $\mathrm{R}$. & 0030 & O082 & & Sipka Jr. & S. & P53 & & & \\
\hline Roukema & J.A. & 0096 & 0097 & & Sixma & H.J. & 0096 & & & \\
\hline Rücker & M. & 0031 & & & Skourtis & Ch. & P33 & & & \\
\hline Ruff & C. & O195 & & & Slodkowski & M.S. & P36 & & & \\
\hline Rutkowska & J. & 0081 & 0137 & & Slooff & M.J.H. & 0002 & & & \\
\hline
\end{tabular}




\begin{tabular}{|c|c|c|c|c|c|c|c|c|c|}
\hline Slotwinski & $\mathrm{R}$. & P36 & & & Topaloglu & S.T. & 0037 & & \\
\hline Sluijter & B.J.R. & 0179 & & & Tournoys & A. & 0140 & & \\
\hline Sluiter & W. & 0190 & & & Tran & K.T.C. & $\mathrm{O} 213$ & $\mathrm{O} 214$ & \\
\hline Slupecka & M. & P43 & & & Tran & T.C.K. & 0130 & O131 & \\
\hline Smeets & H.J. & 0067 & & & Tribulova & S. & 0161 & & \\
\hline Smeets & $\mathrm{N}$ & O041 & & & Troskie & C.G. & O085 & & \\
\hline Smith & A. & 0007 & 0061 & & Truijers & M. & O162 & & \\
\hline Smitten von & $\mathrm{K}$. & 0153 & & & Tsembeli & A. & P18 & & \\
\hline Smit-van Oosten & A. & 0136 & & & Tsivian & M. A. & 0033 & O092 & \\
\hline Smyrnis & A. & 0126 & P17 & P18 P48 & Tsunoda & S. & O174 & & \\
\hline Sokolis & D.P. & 0062 & & & Tufan & $\mathrm{T}$. & 0036 & & \\
\hline Somers & S. & O069 & & & Tukamoto & S. & 0013 & P02 & \\
\hline Sorg & $\mathrm{H}$. & 0156 & & & Tuncel & P. & 0104 & & \\
\hline Sosef & M.N. & O207 & & & Turkyilmaz & S.T. & 0037 & & \\
\hline Sozuer & E. & $\mathrm{P} 15$ & P19 & P35 & Ucar & D. & 0038 & & \\
\hline Spagnoli & G.C. & 0182 & 0183 & & Uchinami & H.U. & 0093 & & \\
\hline Spiegel & H.U. & 0127 & 0189 & & Uemoto & S.U. & P38 & P50 & \\
\hline Spigelman & A.D. & 0086 & & & Uludag & O. & O124 & 0143 & \\
\hline Spijkers & K.J. & 0096 & & & Unal & E. & P23 & & \\
\hline Sridhar & $\mathrm{R}$. & 0014 & & & Unlu & S.M. & 0038 & & \\
\hline Stachyra & E. & 0081 & & & Utebey & G. & 0090 & & \\
\hline Stam & H.J. & $\mathrm{O} 215$ & & & Uyama & $\mathrm{N}$ & P14 & P38 & \\
\hline Stamatakos & M. & P17 & P18 & & Valente & M.L. & 0070 & & \\
\hline Stanczyk & M. & 0176 & & & Valente & M.V. & 0017 & & \\
\hline Stassen & L.P.S. & 0050 & 0129 & 0132 & Vanhorebeek & I. & O202 & O206 & \\
\hline Stelmach & E. & O064 & 0098 & & Vanizor Kural & B.V.K. & 0037 & & \\
\hline Stene & C. & $\mathrm{O} 217$ & & & Váradi & $\mathrm{R}$. & 0184 & & \\
\hline Steyerberg & E.W. & 0050 & & & Vardar & E. & P10 & & \\
\hline Stijn van & M..FM. & 0171 & & & Varga & G. & O184 & & \\
\hline Stoel van der & A. & $\mathrm{O} 025$ & & & Varga & R. & O186 & & \\
\hline Stoep van der & A. & 0083 & & & Varin & $\mathrm{N}$. & 0046 & & \\
\hline Stratos & I. & 0030 & O082 & & Vasilakos & D. & P33 & & \\
\hline Supe & A. & 0043 & & & Vatansev & C. & 0035 & & \\
\hline Svarvar & C. & 0153 & & & Vecchiato & M. & 0070 & & \\
\hline Swart & S. & 0009 & & & Vécsei & $\mathrm{L}$. & 0118 & & \\
\hline Swiatek & M. & 0137 & & & Veen van & R.N. & 0149 & & \\
\hline Swoboda & E. & 0064 & & & Velde van de & C.J.H. & 0096 & O097 & \\
\hline Syed & F. & O039 & & & Velden van der & A.W. & O219 & & \\
\hline Szabó & A. & 0120 & O158 & 01860135 & Verdaasdonk & E.G.G. & 0129 & 0132 & \\
\hline Szabolcs & Z. & 0135 & & & Veres & $\mathrm{G}$. & 0135 & & \\
\hline Szczesny & G. & 0081 & & & Verhoef & C. & $\mathrm{O} 210$ & 0125 & 0147 \\
\hline Tada & M.T. & P38 & P50 & & Verhoeven & E.L.G. & 0056 & & \\
\hline Takacs & I.E. & P54 & & & Verkade & H.J. & 0002 & & \\
\hline Takada & H.T. & P30 & & & Vermaas & M. & 0147 & & \\
\hline Takagi & $\mathrm{H}$. & P31 & P32 & & Vermeulen & C. & O159 & O181 & \\
\hline Takahashi & S. & 0162 & & & Verweij & M. & O203 & & \\
\hline Takahashi & T.T. & 0093 & & & Vetelainen & R.L. & 0185 & & \\
\hline Tamaki & N.T. & P38 & & & Vilar & J. & 0163 & 0006 & \\
\hline Tanaka & E. & 0174 & & & Vincenzo Bondì & M.S. & 0144 & & \\
\hline Tanaka & E.T & P08 & & & Virich & G. & O197 & & \\
\hline Tanaka & H.T. & P30 & & & Visser & D.S. & 0002 & & \\
\hline Taneri & F. & P24 & P25 & & Vliet van & A.K. & 0100 & 0110 & 01190185 \\
\hline Tang & B. & 0053 & & & Vogels & L.M.M. & 0083 & & \\
\hline Tatkan & Y. & 0011 & & & Vollmar & B. & 0001 & 0030 & 008200940141 \\
\hline Tatlicioglu & $\mathrm{E}$. & 0105 & P37 & P40 & & & 0156 & & \\
\hline Teerlink & $\mathrm{T}$. & O202 & & & Vries de & E.E. & O057 & & \\
\hline Teijink & JA.W. & 0164 & & & Vrijland & W.W. & 0149 & & \\
\hline Tekin & A. & 0035 & & & Wadoodi & A. & 0061 & & \\
\hline Tempel & D. & 0163 & 0006 & & Waeckel & $\mathrm{L}$. & 0163 & 0006 & \\
\hline Terzi & C. & 0040 & & & Wagh & $\mathrm{R}$. & 0111 & & \\
\hline Testini & M. & 0087 & & & Wagner & S. & 0161 & & \\
\hline Tezel & $\mathrm{E}$. & P27 & & & Wal van der & J.B.C. & 0149 & O190 & $\mathrm{O} 214$ \\
\hline Tha-In & $\mathrm{T}$. & 0020 & & & Walpoth & B.H. & 0133 & & \\
\hline Thomas & M. & 0009 & & & Waltham & M. & 0061 & & \\
\hline Theocharis & S. & 0122 & & & Wang & Y. & O204 & & \\
\hline Thompson & M.M. & 0021 & 0052 & & Watanabe & $\mathrm{G}$. & 0054 & 0071 & P31 \\
\hline Thorlacius & H. & O193 & O205 & $\mathrm{O} 220$ & Weber & F.W. & $\mathrm{O} 023$ & $\mathrm{O} 024$ & \\
\hline Thornberg & C. & 0193 & P52 & & Weber & $\mathrm{G}$. & P42 & & \\
\hline Tiel van & S. & 0159 & & & Weber & W.P. & 0182 & 0183 & \\
\hline Tielliu & I.F.J. & O056 & & & Wegen van der & P.G. & O029 & & \\
\hline Tigchelaar-Gutter & $\mathrm{W}$ & O076 & & & Wei & $\mathrm{L}$. & 0142 & & \\
\hline \multirow{2}{*}{ Tilanus } & H.W. & 0019 & 0020 & O029 O212 O215 & Wei & W.L. & 0139 & & \\
\hline & & $\mathrm{O} 216$ & O219 & & Weidema & W.F. & 0050 & & \\
\hline Tol van den & M.P. & 0112 & 0115 & & Weigang & E. & 0135 & & \\
\hline Tolba & $\mathrm{R}$. & 0139 & 0142 & & Weijde van der & J. & $\mathrm{O} 212$ & & \\
\hline Tongeren van & R.B.M. & 0160 & & & Weijden van der & $\mathrm{T}$. & 0096 & O097 & \\
\hline
\end{tabular}




\begin{tabular}{|c|c|c|c|c|}
\hline Weimar & W. & O130 & O131 & $\mathrm{O} 213$ \\
\hline Welten & R.J.Th.J. & O164 & & \\
\hline Wentink & $\mathrm{N}$ & 0130 & 0131 & O213 O214 \\
\hline Wettstein & R. & 0005 & & \\
\hline Wiel-Ambagts- & & & & \\
\hline heer aan de & G. & O159 & & \\
\hline Wiggers & T. & 0025 & & \\
\hline Wijnands & P.G.J.T.B & 0179 & & \\
\hline Wilt de & J.H.W. & O125 & O147 & O210 \\
\hline Winkel & T.A. & P55 & & \\
\hline Winkler & T. & 0030 & & \\
\hline Witherow & H. & O195 & & \\
\hline Witteman & B.P.L. & O057 & & \\
\hline Wittgren & L. & P52 & & \\
\hline Wluka & A. & O079 & & \\
\hline Wolinski & J. & P43 & & \\
\hline Wunnik & B. & O124 & & \\
\hline Yacoub & W. & 0069 & & \\
\hline Yagci & G. & 0036 & & \\
\hline Yamada & E. & P32 & & \\
\hline Yamamoto & Y.Y. & 0091 & O093 & O142 \\
\hline Yanagida & A.Y. & P38 & & \\
\hline Yasuchika & K. & P50 & & \\
\hline Yazicioglu & O. & P24 & & \\
\hline Yenisey & C. & P10 & & \\
\hline Yilmaz & F. & P11 & & \\
\hline Yilmaz & $\mathrm{T}$. & P37 & & \\
\hline Yilmaz & N. & P15 & P19 & P35 \\
\hline Yilmaz & Z.Y. & O201 & P41 & \\
\hline Yilmazer & D. & 0090 & & \\
\hline Yomoyuki & S. & 0013 & & \\
\hline Yoritaka Nakano & Y.N. & O088 & & \\
\hline Yoshimoto & $\mathrm{T}$. & P39 & & \\
\hline Yoshioka & M.Y. & 0091 & O093 & \\
\hline You & D. & 0163 & 0006 & \\
\hline Yüksel & O. & 0004 & O105 & \\
\hline Yukselen & V. & P10 & & \\
\hline Yurci & A. & P19 & & \\
\hline Yuzer & Y. & P11 & & \\
\hline Zaleska & M. & O079 & & \\
\hline Zaleska & Z.M. & P36 & & \\
\hline Zandvoort & J.H. & 0136 & & \\
\hline Zaninotto & G. & O187 & & \\
\hline Zdzalik & D. & 0098 & & \\
\hline Zeebregts & C.J. & 0056 & & \\
\hline Zeytunlu & M. & P11 & & \\
\hline Zonderhuis & B. & P56 & & \\
\hline Zoucas & E. & O169 & & \\
\hline Zundert van & A. & O028 & & \\
\hline
\end{tabular}

\section{7 \\ HAEMORRHOIDAL ARTERY LIGATION OPERATION - A FIRST LINE TREATMENT ?}

M. Chand, P.J. Moore, T. Andrews, G.F. Nash, A.D. Clarke

Poole Hospital, Poole, United Kingdom

Introduction: Doppler guided haemorrhoidal artery ligation operation (HALO) is a recent definitive surgical intervention for bleeding haemorrhoids, which can be performed with or without general anaesthesia. The most common therapy for piles is perhaps rubber band ligation (RBL) however recent evidence suggests it is poorly tolerated by most patients. We investigated the different satisfaction outcomes after HALO compared to the common first line treatment of rubber band ligation (RBL)

Methods: Patients were divided into 3 groups. Group A consisted of patients who had undergone banding only. Group B consisted of patients who had undergone banding and then HALO, and group $\mathrm{C}$ consisted of patients who had undergone HALO with no previous interventions. Retrospective data from a single centre was collected using a structured questionnaire relating to symptoms, bleeding, pain, complications and satisfaction.

Results: A total number of 45 patients were recruited with 15 patients in each group. All patients recorded their major symptom to be bleeding. Bleeding and pain were measured on a visual analogue scale (0-4) and satisfaction was measured on a visual analogue scale (0-3). Pain was scored at day 2 post-procedure while all other outcomes were measured at 6 weeks.

Group A, as compared to both Groups B and C, had statistically significant higher bleeding and pain scores, and lower satisfaction scores ( $p$-values are shown in the table below). However, there was no significant difference between those patients who had undergone HALO as a first-line intervention and those following failed RBL.

$\begin{array}{lcccc}\text { Outcomes } & \text { A } & \text { B } & \text { C } & \text { p-value } \\ \text { Bleeding } & 1.133 & 0.200 & 0.800 & \& \mid \mathrm{tt} ; 0.001 \\ \text { Pain } & 0.700 & 0.066 & 0.266 & \& \mathrm{It} ; 0.001 \\ \text { Satisfied } & 1.466 & 2.500 & 2.800 & \& \mathrm{It} ; 0.001\end{array}$

There were no reported significant complications at follow up and all patients were discharged on the day of the procedure.

Conclusion: The outcomes and satisfaction rates of patients undergoing HALO were significantly higher than those having undergone RBL alone. We propose that in fit and willing patients with bleeding haemorrhoids, HALO may be suggested as a definitive first-line procedure rather than be offered only after failed banding.

\section{2 \\ GENETIC TESTING IN PHEOCHROMOCYTOMA; TOOLS FOR CLINICAL PRACTICE}

B.J. Petri, C.H.J. van Eijck, E. van der Harst, W.W. de Herder, W.N.M. Dinjens, R.R. de Krijger

Erasmus MC University Rotterdam, Rotterdam, The Netherlands

Pheochromocytomas (PCC) are tumors occurring in the adrenal medulla . In $10 \%$ they are bilateral, in $10 \%$ they are malignant, and in $10 \%$ they occur outside the adrenal, and are called sympathetic paragangliomas (sPGL). Approximately $24 \%$ of these tumors appear in familial tumor syndromes, the remaining group is apparently sporadic (76\%). Several causative genes for familial cancer syndromes are known, including the RET gene causing Multiple Endocrine Neoplasia (MEN), the VHL gene causing Von Hippel Lindau disease (VHL), the SDHB, $-\mathrm{C}$, and â $\mathfrak{E}^{\prime} \mathrm{D}$ genes causing PheochromocytomaParaganglioma syndromes, and the NF1 gene causing Neurofibromatosis type I (NF1). Tumorigenesis of apparently sporadic PCC is not well understood. In clinical practice most familial tumor syndromes are well-recognized by their additional symptoms and signs, but they may be difficult to diagnose when the patient has a negative family history and a PCC is the first clinical sign. Therefore we reviewed the literature to set up a flow chart to help and inform the patients with PCC optimally. We suggest that every patient, younger than 50 years with a PCC, no signs of neurofibromatosis and a negative family history, should undergo genetic testing. In case of apparently sporadic PCC we suggest to test for all before mentioned genes, except the NF1 gene, because of the clear clinical presentation. In case of bilateral PCC we suggest to test first the RET and the VHL genes, and if these are nomal to test the SDHB, and â€'D genes. In case of SPGL we suggest to test SDHB, and $\hat{a} \in$ ' $D$ in first instance, when negative the VHL and the RET genes. When a germline mutation is found the clinician can screen for other entities in the various familial tumor syndromes to detect other manifestation of the disease. 